\title{
The NPOESS Preparatory Project: Mission Concept and Status
}

\author{
Robert E Murphy ${ }^{1}$, Raynor Taylor ${ }^{2}$, Daniel S DeVito², Janice K Smith ${ }^{2}$, Joy Henegar², \\ James C Dodge ${ }^{3}$, Peter Wilczynski ${ }^{4}$, Michael Kelly ${ }^{4}$, Stanley Schneider ${ }^{4}$, Carol Welsch ${ }^{4}$, \\ Hal Bloom ${ }^{4}$, Stephen A Mango ${ }^{4}$, Michael Crison ${ }^{5}$ \\ ${ }^{1}$ NASA Goddard Space Flight Center, Greenbelt, MD, USA \\ Tel: 301 614-5827. e-mail:rmurphy@LTPMail.gsfc.nasa.gov \\ ${ }^{2}$ NASA Goddard Space Flight Center, Greenbelt, MD, USA \\ ${ }^{3}$ NASA Headquarters, Washington, DC, USA \\ ${ }^{4}$ The Integrated Program Office, National Polar-Orbiting Operational Environmental Satellite System, \\ Silver Spring, MD, USA \\ ${ }^{5}$ NOAA NESDIS, Silver Spring, MD, USA
}

\begin{abstract}
NPP is a joint NASA/IPO mission to extend selected systematic measurements initiated by the Terra and Aqua missions and to proovide risk reduction for NPOESS. The key sensor properties and mission features are summarized.
\end{abstract}

\section{INTRODUCTION}

In 2005 NASA and the Integrated Program Office (IPO) for the National Polar-orbiting Operational Environmental Satellite System (NPOESS) will conduct a joint mission to extend a number of environmental measurement series initiated with NASA's Terra and Aqua missions, and to provide risk reduction for three critical NPOESS sensors. This joint mission will launch the NPOESS Preparatory Project (NPP) satellite carrying a moderate resolution imager and a sensor suite to acquire atmospheric profiles of temperature and water vapor.

NPP will be placed into a sun synchronous orbit at $824 \mathrm{~km}$ with a descending node at 10:30 AM. This orbit will provide a 16-day (quasi 8-day) ground track repeat and full global coverage by the imager in a single day. The orbit closely matches the groundtrack and repeat cycle of the Terra and Aqua missions.

\section{SENSORS}

NPP will carry the Visible Infrared Imaging Radiometer Suite (VIIRS), a multispectral imager with 22 spectral bands. VIIRS will acquire data to determine a wide variety of properties of the land and ocean surface as well as of cloud and aerosol properties. There are three types of spectral bands; 5 high resolution $(371 \mathrm{~m}$ at nadir) for imagery (manually interpreted) studies, 16 radiometric bands with medium resolution $(742 \mathrm{~m}$ at nadir), and one "day-night" band to provide imagery across the
Table 1 NPP MISSION SUMMARY

\begin{tabular}{|ll|}
\hline Sensors: & \\
\hline VIIRS & 22 Bands \\
(Imaging & $.4-12 \mu$ \\
Radiometer) & $372 \& 744 \mathrm{~m}$ at Nadir \\
\hline CrIS & $1142 \mathrm{Channels}$ \\
(IR Interferometer & $3.9-15.4 \mathrm{~m}$ \\
Sounder) & $14 \mathrm{~km}$ at Nadir \\
\hline ATMS & $22 \mathrm{Channels}$ \\
(Microwave Sounder) $23.8-183 \mathrm{GHz}$ \\
& $2.2^{\circ} \& 5.5^{\circ}$ at Nadir \\
\hline Launch/Mission & $2005 / 5$ years \\
Life & $824 \mathrm{~km}$ polar sun-sync \\
\hline Orbit: & $10: 30$ AM descending node \\
\hline Data Products & \\
\hline IPO IDPS & Pre-Operational \\
\hline NASA SDS & Products (EDRs) \\
\hline
\end{tabular}

terminator. The imagery bands have high radiometric fidelity and will be used in concert with the radiometric bands to produce data products. There are 9 bands between .4 and $1 \mu$. Six of them have dual gains, permitting their use over ocean and land surfaces. Eight bands between 1.2 and $4 \mu$ are used for surface, cloud and aerosol measurements. Four bands cover the long wavelength region between 8.55 and $12 \mu$ for use in imagery, land and sea surface temperature and fire detection.

A more complete description of VIIRS is given in presentations by Welsch et al. (A NextGeneration Operational Sensor for NPOESS: The VIIRS (Visible Infrared Imaging Radiometer Suite)) in the Instrumentation and Future technologies session and by Murphy et 
al.(Using VIIRS to Provide Data Continuity with MODIS) in the EOS Terra session.

VIIRS will extend the measurement series initiated by the MODerate Resolution Imaging Spectroradiometer (MODIS) on Terra. VIIRS is being built by Raytheon under contract from the IPO.

NPP will also carry the first copies of the CrossTrack Infrared Sounder (CrIS) and the Advanced Technology Microwave Sounder (ATMS). CrIS/ATMS extends the measurement series that will be initiated by the Atmospheric Infrared Sounder (AIRS), the Advanced Microwave Sounding Unit (AMSU) and the Humidity Sounder Brazil (HSB) on Aqua.

CrIS is an interferometer with three spectral bands, 3.9-4.6 $\mu, 5.7-8.3 \mu$, and 9.1-15.4 $\mu$. Each spectral band has an associated interferometer with a $3 \times 3$ detector array. There are 1,142 spectral channels within the 3 spectral bands. CrIS will retrieve high horizontal spatial resolution $(15 \mathrm{~km}$ at nadir) temperature profiles in layers of $1 \mathrm{~km}$ in the lower troposphere to an accuracy of better than $1^{\circ}$ for clear to partly cloudy pixels. It is also capable of determining column abundances of a variety of trace gases. The $3 \times 3$ array of detectors in each of the 3 interferometers yields an overall footprint of 45 $\mathrm{km}$ that is co-registered to the ATMS footprints. It is being built by ITT under contract from the IPO. A more complete description of CrIS is given by Bloom (The Cross-Track Infrared Sounder (CRIS): A Sensor for Operational Meteorological) in the Atmosphere and Climate session

ATMS is a cross track scanning microwave radiometer with 22 channels. Long wavelength channels at 23.8 and $31.4 \mathrm{GHz}$ are used for tropospheric water vapor. There are 11 channels from 51.76 to $57.6 \mathrm{GHz}$ that are used for temperature profiles. Channels at $89 \mathrm{GHz}, 166$ $\mathrm{GHz}$ and 5 more at $183 \mathrm{GHz}$ complete the water vapor profiling. Spatial resolution ranges from $5.5^{\circ}$ to $2.2^{\circ}$. ATMS is being built by Aerojet under contract from NASA.

CrIS and ATMS provide a single set of data products. When cloud conditions permit (typically less than $50 \%$ cloud cover within any of the 9 CrIS footprints) data from both sensors will be used. When cloudiness exceeds the threshold, ATMS alone will be used to produce the products at reduced accuracies.

\section{DATA PRODUCTS}

The Interface Data Processing Segment (IDPS) of the IPO will produce a stream of 29 environmental data records (EDRs) for use by the operational users in the Department of Defense and the Department of Commerce. EDRs are roughly equivalent to NASA level 2 products. These data products will be produced in near real time. See table 2.

Later in 2001 NASA will solicit proposals from the global change science community to develop algorithms and produce long-term consistent data sets using the sensors on NPP. To the maximum extent possible NASA will rely on the EDRs produced by the IDPS. However, proposals to further develop the algorithms to address key scientific questions (see Exploring Our Home Planet, Earth Science Enterprise

Table 2 NPP DATA PRODUCTS

\begin{tabular}{|ll|}
\hline PRODUCT NAME & SENSOR \\
\hline Imagery (Manually Produced) & \\
Cloud Cover & VIIRS \\
Cloud Type & VIIRS \\
Ice Edge Location & VIIRS \\
Ice Concentration & VIIRS \\
\hline Atmosphere & \\
Vertical Moisture Profile & CrIS/ATMS \\
Vertical Temperature Profile & CrIS/ATMS \\
Vertical Pressure Profile & CrIS/ATMS \\
Aerosol Optical Thickness & VIIRS \\
Aerosol Particle size & VIIRS \\
Suspended Matter & VIIRS \\
\hline Clouds & \\
Cloud Cover/layers & VIIRS \\
Cloud Effective Particle Size & VIIRS \\
Cloud Optical Thickness & VIIRS \\
Cloud Top Height & VIIRS \\
Cloud Top Pressure & VIIRS \\
Cloud Top Temperature & VIIRS \\
Cloud Base Height & VIIRS \\
\hline Land & \\
Albedo (Surface) & VIIRS \\
Vegetation Index & VIIRS \\
Surface Type & VIIRS \\
Land Surface Temperature & VIIRS \\
Snow Cover & VIIRS \\
Soil Moisture & VIIRS \\
Active Fires & VIIRS \\
\hline Ocean/Water/Ice & \\
Sea Surface Temperature & VIIRS \\
Ocean Color/Chlorophyll & VIIRS \\
Sea Ice Age/Edge & VIIRS \\
Ice Surface Temperature & VIIRS \\
Net Heat Flux & VIIRS \\
\hline
\end{tabular}


Strategic Plan, NASA, 2001) will be considered. These level 2, 3 and 4 products, which are known as Climate Data Records (CDRs), will include multi-date data composites such as weekly cloud-cleared surface properties. Research quality level 1 products will also be made.

The NASA CDRs will be produced in the Science Data Segment (SDS). CDRs will not be produced in near real time and will make optimal use of ancillary data. Periodic reprocessing of the key data products will provide consistent longterm data sets critical to global change research. Advanced algorithms will be incorporated into the operational data production stream to the extent that they are compatible with operational needs.
The IPO EDRs and NASA CDRs will be available to the global change and applications user communities through an Archive and Distribution Segment (ADS) to be developed by NOAA.

\section{SPACECRAFT}

The NPP spacecraft will be selected in early 2002. All data will be kept in on-board storage for transmission to a ground station in Svalbaard, Norway. In addition, all data will be available to users via direct broadcast at no charge. A 3meter class $\mathrm{X}$-band receiving system is required. The spacecraft will support a full range of calibration maneuvers, including periodic deep space views by all sensors. 